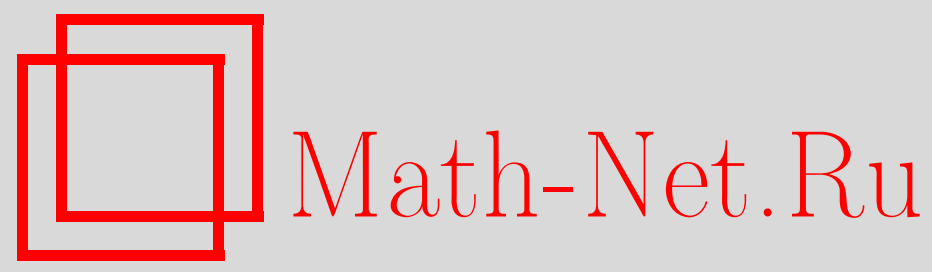

Г. Г. Лаптев, Априорные оценки сильных решений полулинейных параболических уравнений, Матем. заметки, 1998, том 64, выпуск 4, 564-572

DOI: https://doi.org/10.4213/mzm1431

Использование Общероссийского математического портала Math-Net.Ru подразумевает, что вы прочитали и согласны с пользовательским соглашением http://www . mathnet.ru/rus/agreement

Параметры загрузки:

IP : 52.205 .19 .152

26 апреля 2023 г., 12:34:11

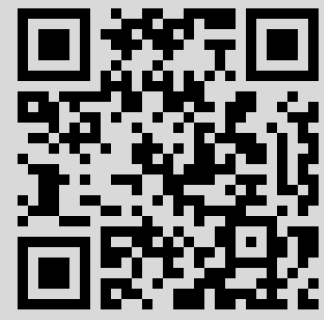




\section{АПРИОРНЫЕ ОЦЕНКИ СИЛЬНЫХ РЕШЕНИЙ ПОЛУЛИНЕЙНЫХ ПАРАБОЛИЧЕСКИХ УРАВНЕНИЙ}

\section{Г.Г. Лаптев}

Рассматривается начально-краевая задача для полулинейного параболического уравнения

$$
\frac{\partial u}{\partial t}+\sum_{|\alpha| \leqslant 2 b} a_{\alpha}(x, t) D^{\alpha} u=f\left(x, t, u, D u, \ldots, D^{2 b-1} u\right)
$$

где левая часть - линейный равномерно параболический оператор порядка $2 b$. Устанавливаются достаточные условия роста функции $f$ по переменным $u, D u, \ldots, D^{2 b-1} u$, при которых имеет место априорная оценка нормы решения в пространстве Соболева $W_{p}^{2 b, 1}$ через младшую норму в пространстве Лебега суммируемых функций $L_{l, m}$.

Библиограффия: 8 названий.

Введение. Изучается начально-краевая задача для полулинейного параболического уравнения, имеющего высокий порядок по пространственным переменньм и первьй по времени. Рассматривается проблема существования априорных оценок решения в пространстве Соболева через его норму в пространстве Лебега. Определяются показатели соответствующих нелинейностей, при которых эта проблема имеет решение. Используемый в работе интерполящионньй метод получения априорных оценок применительно к эллиптическим уравнениям развивался С.И. Похожаевым в работах [1], [2]. Там же были построены контрпримеры, показывающие неулучшаемость показателей нелинейностей.

1. Постановка задачи и основной результат. Пусть $a \in \mathbb{R}_{+} \equiv\{a \in \mathbb{R}: a \geqslant 0\}$. Введем следующие обозначения:

$$
\begin{aligned}
& x=\left(x_{1}, \ldots, x_{N}\right) \text { - точка в пространстве } \mathbb{R}^{N} ; \\
& \Omega \subset \mathbb{R}^{N}-\text { ограниченная область с границей } \partial \Omega \text { класса } C^{2 b}, b \geqslant 1 ; \\
& Q_{t}=\Omega \times(a, a+t) \text { - цилиндрическая область в пространстве } \mathbb{R}^{N+1}, t \in \mathbb{R}_{+} ; \\
& \partial Q_{t}=\partial \Omega \times(a, a+t) \text { - боковая поверхность цилиндра } Q_{t} ; \\
& Q \equiv Q_{T}=\Omega \times(0, T) \text { - цилиндр заданной высоты } T>0 .
\end{aligned}
$$

Всюду далее функции считаются вещественнозначньпи. В работе используются следующие функциональные пространства [3, с. 126], [4, с. 118]:

пространство суммируемых функций $L_{p}\left(Q_{t}\right), p \geqslant 1$, с нормой

$$
\|u\|_{p ; Q_{t}}=\left(\int_{a}^{a+t} \int_{\Omega}|u(x, t)|^{p} d x d t\right)^{1 / p}
$$

Работа выполнена при финансовой поддержке Российского фонда фундаментальных исследований, грант № 96-15-96102. 
анизотропное пространство суммируемых функций $L_{q, r}\left(Q_{t}\right), q, r \geqslant 1$, с нормой

$$
\|u\|_{q, r ; Q_{t}}=\left(\int_{a}^{a+t}\left(\int_{\Omega}|u(x, t)|^{q} d x\right)^{r / q} d t\right)^{1 / r}
$$

анизотропное пространство Соболева $W\left(Q_{t}\right) \equiv W_{p}^{2 b, 1}\left(Q_{t}\right)$ с нормой

$$
\|u\|_{W\left(Q_{t}\right)}=\|u\|_{p ; Q_{t}}+\sum_{i=1}^{N}\left\|\frac{\partial^{2 b} u}{\partial x_{i}^{2 b}}\right\|_{p ; Q_{t}}+\left\|\frac{\partial u}{\partial t}\right\|_{p ; Q_{t}} .
$$

Через $D^{i} u$ обозначим вектор из частных производных $D^{\alpha} u$ функции $u(x, t), \alpha=\left(\alpha_{1}, \ldots\right.$, $\left.\alpha_{N}, 0\right),|\alpha|=i$. Этот вектор имеет $N_{i}$ компонент, где $N_{i}$ - число различных мультииндексов $\alpha=\left(\alpha_{1}, \ldots, \alpha_{N}, 0\right),|\alpha|=i$.

Работа посвящена изучению обобщенных решений $u \in W(Q)$ задачи

$$
\begin{gathered}
\frac{\partial u}{\partial t}+\sum_{|\alpha| \leqslant 2 b} a_{\alpha}(x, t) D^{\alpha} u=f\left(x, t, u, D u, \ldots, D^{2 b-1} u\right), \\
\left.u\right|_{t=0}=0,\left.\quad D^{\omega} u\right|_{\partial Q}=0, \quad|\omega| \leqslant b-1,
\end{gathered}
$$

где $a_{\alpha}(x, t)$ - заданные достаточно гладкие функции.

Предполагаются выполненными следующие условия:

1) линейньй оператор

$$
\frac{\partial u}{\partial t}+\sum_{|\alpha| \leqslant 2 b} a_{\alpha}(x, t) D^{\alpha} u
$$

является равномерно параболическим [4];

2) функция $f\left(x, t, \xi_{0}, \ldots, \xi_{2 b-1}\right)$ определена на $\bar{Q} \times \mathbb{R} \times \mathbb{R}^{N} \times \cdots \times \mathbb{R}^{N_{2 b-1}}$ и является каратеодориевой, т.е. измеримой по $x, t$ при $\xi_{0}, \ldots, \xi_{2 b-1} \in \mathbb{R} \times \mathbb{R}^{N} \times \cdots \times \mathbb{R}^{N_{2 b-1}}$ и непрерьвной по $\xi_{0}, \ldots, \xi_{2 b-1}$ для почти всех $x, t \in Q$;

3 ) при $x, t, \xi_{0}, \ldots, \xi_{2 b-1} \in Q \times \mathbb{R} \times \mathbb{R}^{N} \times \cdots \times \mathbb{R}^{N_{2 b-1}}$ выполнено неравенство

$$
\left|f\left(x, t, \xi_{0}, \ldots, \xi_{2 b-1}\right)\right| \leqslant b(x, t)+\sum_{i=0}^{2 b-1} b_{i}(x, t)\left|\xi_{i}\right|^{\mu_{i}},
$$

где $b(x, t) \in L_{p}(Q), b_{i}(x, t) \in L_{q_{i}, r_{i}}(Q)$;

4) $p>N+2 b, q_{i} \geqslant p, p \leqslant r_{i}<\infty, i=0, \ldots, 2 b-1$;

5) степени $\mu_{i}$ определяются формулой

$$
\mu_{i}=\frac{1}{i+N / l+2 b / m}\left(2 b+\frac{N}{l}+\frac{2 b}{m}-\frac{N}{q_{i}}-\frac{2 b}{r_{i}}\right),
$$

где $1 \leqslant l, m \leqslant \infty$ - некоторые числа;

6) вьполнены неравенства

$$
\mu_{i} \frac{p q_{i}}{q_{i}-p} \geqslant l, \quad \mu_{i} \frac{p r_{i}}{r_{i}-p} \geqslant m, \quad i=0, \ldots, 2 b-1 ;
$$

из 4) следует, что всегда $\mu_{i}>1$.

Основным утверждением работы является 
ТЕОРема 1. Пусть выполнены условия 1)-6) и для любого возмохсного решения задачи (1) известна априорная оченка $\|u\|_{l, m ; Q} \leqslant M$. Тогда

$$
\|u\|_{W_{p}^{2 b, 1}(Q)} \equiv\|u\|_{W(Q)} \leqslant C
$$

где константа С зависит только от известных данных.

ЗАмЕчАниЕ. Степени $\mu_{i}$ в условии 5$)$ аналогичны соответствующим степеням из работы [1], однако в отличие от рассмотренных там эллиптических уравнений для параболических задач эти степени достигаются, что показывает теорема 1 . Этот факт впервые был отмечен в работах [5], [6], где изучались случаи $l=2$ и $l=\infty$ для $m=q_{i}=r_{i}=\infty$. При этом на функцию $f\left(x, t, \xi_{0}, \ldots, \xi_{2 b-1}\right)$ накладывались дополнительные условия гладкости.

2. Доказательство основного результата. В основе доказательства теоремы 1 лежат следующие два момента: использование интерполяционного неравенства для оценки $\left\|D^{i} u\right\|^{\mu_{i}}$ через $\|u\|_{W\left(Q_{t}\right)}$ и $\|u\|_{l, m ; Q_{t}}$ и применение теоремы о разрешимости линейных параболических задач в малом цилиндре [4, с. 120]. С помощью специальных построений показано, что коэффициенты при $\|u\|_{W\left(Q_{t}\right)}$ не зависят от высоты цилиндра, когда эта высота мала. Это позволяет за счет использования цилиндров малой высоты установить достижимость предельньх степеней $\mu_{i}$, которые не достигаются в эллиптическом случае.

ЛЕмма 1. Пусть $u \in W\left(Q_{\tau}\right), Q_{\tau}=\Omega \times\left(t_{0}, t_{0}+\tau\right) \subset Q$. Пусть $u\left(x, t_{0}\right)=0 u$ выполнены условия 4)-6). Тогда

$$
\left\|D^{i} u\right\|_{z_{x i}, z_{t i} ; Q_{\tau}} \leqslant c_{0}\|u\|_{W\left(Q_{\tau}\right)}^{1 / \mu_{i}}\|u\|_{l, m ; Q_{\tau}}^{1-1 / \mu_{i}}+c_{0}\|u\|_{l, m ; Q_{\tau}}
$$

$2 \partial e$

$$
z_{x i}=\frac{\mu_{i} p q_{i}}{q_{i}-p}, \quad z_{t i}=\frac{\mu_{i} p r_{i}}{r_{i}-p},
$$

причем константа с $c_{0}$ не зависит от $t_{0} u \tau$.

ДокАЗАТЕЛЬСТво. Согласно условию леммы функция $u(x, t)$ первоначально задана в цилиндре малой высоты $\tau$. Продолжим ее на весь цилиндр $Q$, доопределив нулем на отрезке $\left[0, t_{0}\right]$, а затем отобразив четным образом относительно точки $t_{0}+\tau$. Подробнее,

$$
\widetilde{u}(x, t)= \begin{cases}0, & t \leqslant t_{0}, \\ u(x, t), & t_{0}<t<t_{0}+\tau, \\ u\left(x, 2\left(t_{0}+\tau\right)-t\right), & t_{0}+\tau \leqslant t<t_{0}+2 \tau, \\ 0, & t \geqslant t_{0}+2 \tau .\end{cases}
$$

Заметим, что согласно теореме о следах [4, с. 116] при каждом $t \in\left[t_{0}, t_{0}+\tau\right]$ определен след $u(x, t)$ как непрерьвная в $\bar{\Omega}$ функция. В частности, вполне определены функции $u\left(x, t_{0}\right)$ и $u\left(x, t_{0}+\tau\right)$. Очевидно, $\widetilde{u} \in W(Q)$, при этом

$\|\widetilde{u}\|_{l, m ; Q} \leqslant 2\|u\|_{l, m ; Q_{\tau}}, \quad\|\widetilde{u}\|_{W(Q)} \leqslant 2\|u\|_{W\left(Q_{\tau}\right)}, \quad\left\|D^{i} \widetilde{u}\right\|_{z_{x i}, z_{t i} ; Q} \geqslant\left\|D^{i} u\right\|_{z_{x i}, z_{t i} ; Q_{\tau}}$. 
$\mathrm{K}$ функции $\widetilde{u} \in W(Q)$ применим теорему вложения анизотропных пространств Соболева [7], [3, с. 304], согласно которой найдется $\delta_{0} \in \mathbb{R}_{+}$такое, что для любого $0<\delta \leqslant \delta_{0}$

$$
\left\|D^{i} \widetilde{u}\right\|_{z_{x i}, z_{t i} ; Q} \leqslant C \delta^{-\varkappa_{0}}\|\widetilde{u}\|_{l, m ; Q}+C \delta^{\varkappa_{1}}\left(\sum_{j=1}^{N}\left\|\frac{\partial^{2 b} \widetilde{u}}{\partial x_{j}^{2 b}}\right\|_{p ; Q}+\left\|\frac{\partial \widetilde{u}}{\partial t}\right\|_{p ; Q}\right) .
$$

Здесь

$$
\begin{aligned}
& \varkappa_{0}=\frac{1}{2 b}\left(N\left(\frac{1}{l}-\frac{1}{z_{x i}}\right)+i+2 b\left(\frac{1}{m}-\frac{1}{z_{t i}}\right)\right), \\
& \varkappa_{1}=1-\frac{1}{2 b}\left(N\left(\frac{1}{p}-\frac{1}{z_{x i}}\right)+i+2 b\left(\frac{1}{p}-\frac{1}{z_{t i}}\right)\right) .
\end{aligned}
$$

Отметим также, что константа $C$ не зависит от $t_{0}$ и $\tau$, так как функция $\widetilde{u}$ определена во всем цилиндре $Q$. Возможность применения теоремы вложения при условиях 4)-6) легко проверяется. В частности, $\varkappa_{0}>0, \varkappa_{1}>0$. Далее, положим [3, с. 415]

$$
\delta=\min \left\{\left(\|\widetilde{u}\|_{l, m ; Q}\|\widetilde{u}\|_{W(Q)}^{-1}\right)^{1 /\left(\varkappa_{0}+\varkappa_{1}\right)} ; \delta_{0}\right\} .
$$

В результате несложных преобразований имеем

$$
\left\|D^{i} \widetilde{u}\right\|_{z_{x i}, z_{t i} ; Q} \leqslant C\|\widetilde{u}\|_{W(Q)}^{\varkappa_{0} /\left(\varkappa_{0}+\varkappa_{1}\right)}\|\widetilde{u}\|_{l, m ; Q}^{\varkappa_{1} /\left(\varkappa_{0}+\varkappa_{1}\right)}+C\|\widetilde{u}\|_{l, m ; Q} .
$$

Непосредственный подсчет показьвает, что $\varkappa_{0} /\left(\varkappa_{0}+\varkappa_{1}\right)=1 / \mu_{i}$. Заметим, что степени $\mu_{i}$ выбираются в теореме 1 исходя из этого равенства. Учитывая неравенства (2), получим утверждение леммы.

Лемма 2. Oператор $f[u] \equiv f\left(x, t, u(x, t), \ldots, D^{2 b-1} u(x, t)\right)$ при условиях 2$\left.)-6\right)$ является ограниченным и непрерывным оператором из пространства $W\left(Q_{\tau}\right)$ в $L_{p}\left(Q_{\tau}\right)$ (для любого иилиндра $\left.Q_{\tau}=\Omega \times\left(t_{0}, t_{0}+\tau\right)\right)$.

ДокаЗАТЕЛЬСтво. Так как $p>N+2 b$, по теореме вложения все производные функции $u \in W\left(Q_{\tau}\right)$ по переменным $x_{1}, \ldots, x_{N}$ до порядка $2 b-1$ непрерывны, при этом

$$
\left\|D^{i} u\right\|_{\infty ; Q_{\tau}} \leqslant C\|u\|_{W\left(Q_{\tau}\right)}, \quad i=0, \ldots, 2 b-1 .
$$

Используя 3), получим

$$
\begin{aligned}
\left\|f\left(x, t, u, \ldots, D^{2 b-1} u\right)\right\|_{p ; Q_{\tau}} & \leqslant\|b\|_{p ; Q_{\tau}}+\sum_{i=0}^{2 b-1}\left\|b_{i}\right\|_{p ; Q_{\tau}}\left\|D^{i} u\right\|_{\infty ; Q_{\tau}}^{\mu_{i}} \\
& \leqslant\|b\|_{p ; Q_{\tau}}+\sum_{i=0}^{2 b-1} c\left\|b_{i}\right\|_{q_{i}, r_{i} ; Q_{\tau}}\left(C\|u\|_{W\left(Q_{\tau}\right)}\right)^{\mu_{i}} .
\end{aligned}
$$

Это доказывает ограниченность оператора $f$, откуда согласно общим свойствам оператора суперпозиции следует его непрерьвность. 
ДОКАЗАТЕЛЬСТво ТЕОРЕМЫ 1. Рассмотрим в цилиндре $Q_{\tau}=\Omega \times\left(t_{0}, t_{0}+\tau\right)$ линейную задачу

$$
\begin{gathered}
\frac{\partial u}{\partial t}+\sum_{|\alpha| \leqslant 2 b} a_{\alpha}(x, t) D^{\alpha} u=g(x, t), \\
u\left(x, t_{0}\right)=0,\left.\quad D^{\omega} u\right|_{\partial Q_{\tau}}=0, \quad|\omega| \leqslant b-1 .
\end{gathered}
$$

По условию 1) эта задача является параболической; следовательно, к ней можно применить теорему о разрешимости в малом цилиндре [4, с. 120], согласно которой задача имеет единственное решение $u \in W\left(Q_{\tau}\right)$ для $g \in L_{p}\left(Q_{\tau}\right)$ и

$$
\|u\|_{W\left(Q_{\tau}\right)} \leqslant c_{1}\|g\|_{p ; Q_{\tau}}
$$

причем константа $c_{1}$ не зависит от $t_{0}$ и $\tau$.

Далее, подберем малое $\tau$, исходя из следуюших соображений. Пусть на отрезке $[0, T]$ задана суммируемая функция $\varphi(t)$. Напомним свойство абсолютной непрерывности интеграла Лебега [8, с. 301]: для любого $\varepsilon>0$ найдется $\delta>0$ такое, что для любого измеримого множества $e \subset[0, T]$, удовлетворяющего условию mes $e<\delta$, независимо от его положения выполняется неравенство

$$
\left|\int_{e} \varphi(t) d t\right|<\varepsilon
$$

Применим это утверждение к функциям

$$
\varphi_{i}(t)=\left\|b_{i}(x, t)\right\|_{q_{i} ; \Omega}^{r_{i}}, \quad i=0, \ldots, 2 b-1 .
$$

Напомним, что согласно условию 3 ) считаются конечными нормы

$$
\left\|b_{i}\right\|_{q_{i}, r_{i} ; Q}=\left(\int_{0}^{T}\left\|b_{i}(x, t)\right\|_{q_{i} ; \Omega}^{r_{i}} d t\right)^{1 / r_{i}}
$$

поэтому введенные вьше функции $\varphi_{i}(t)$ суммируемы на $[0, T]$. В качестве множества $e$ выберем отрезки $\left[t_{0}, t_{0}+\tau\right]$. Тогда mes $e=\tau$. Тем самым, свойство абсолютной непрерьвности интеграла Лебега запишется в следующей форме: для любого $\varepsilon>0$ найдется $\tau>0$ такое, что

$$
\left\|b_{i}\right\|_{q_{i}, r_{i} ; Q_{\tau}}=\left(\int_{t_{0}}^{t_{0}+\tau}\left\|b_{i}(x, t)\right\|_{q_{i} ; \Omega}^{r_{i}} d t\right)^{1 / r_{i}}<\varepsilon
$$

независимо от положения точки $t_{0} \in[0, T)$.

Используя изложенное, подберем $\tau$ так, чтобы вьполнялось неравенство

$$
c_{1} \sum_{i=0}^{2 b-1}\left(4 c_{0}\right)^{\mu_{i}} M^{\mu_{i}-1}\left\|b_{i}\right\|_{q_{i}, r_{i} ; Q_{\tau}} \leqslant \frac{1}{2}
$$

где $c_{0}$ - константа из леммы $1, c_{1}$ - константа из неравенства $(3), M$ - постоянная из условия теоремы. Напомним, что они не зависят от выбора $t_{0}$ и $\tau$. 
Разобьем область $Q$ на цилиндры

$Q^{0}=\Omega \times(0, \tau), Q^{1}=\Omega \times(\tau, 2 \tau), \ldots, Q^{k}=\Omega \times(k \tau,(k+1) \tau), \ldots, Q^{K}=\Omega \times(K \tau, T)$

высоты $\tau$. Поскольку $\tau$ фиксировано, число этих цилиндров конечно.

Пусть $u_{0} \in W(Q)$ - решение (1). Рассмотрим задачу (1) в цилиндре $Q^{0}$ как линейную с правой частью $f_{0}=f\left(x, t, u_{0}, \ldots, D^{2 b-1} u_{0}\right)$. Согласно неравенству (3) и условию 3$)$

$$
\begin{aligned}
\left\|u_{0}\right\|_{W\left(Q^{0}\right)} & \leqslant c_{1}\left\|f_{0}\right\|_{p ; Q^{0}}=c_{1}\left\|f\left(x, t, u_{0}, \ldots, D^{2 b-1} u_{0}\right)\right\|_{p ; Q^{0}} \\
& \leqslant c_{1}\left(\|b\|_{p ; Q^{0}}+\sum_{i=0}^{2 b-1}\left\|b_{i}\left|D^{i} u_{0}\right|^{\mu_{i}}\right\|_{p ; Q^{0}}\right) .
\end{aligned}
$$

Для оценки норм в последней сумме применим неравенство Гёльдера с векторными показателями. Имеем

$$
\begin{aligned}
\left\|b_{i}\left|D^{i} u_{0}\right|^{\mu_{i}}\right\|_{p ; Q^{0}} & =\left(\int_{Q^{0}}\left|b_{i}\right|^{p}\left|D^{i} u_{0}\right|^{\mu_{i} p} d x d t\right)^{1 / p} \\
& \leqslant\left(\left\|b_{i}^{p}\right\|_{s_{x i}, s_{t i} ; Q^{0}}\left\|\left|D^{i} u_{0}\right|^{\mu_{i} p}\right\|_{s_{x i}^{\prime}, s_{t i}^{\prime} ; Q^{0}}\right)^{1 / p} \\
& =\left\|b_{i}\right\|_{p_{x i}, p s_{t i} ; Q^{0}}\left\|D^{i} u_{0}\right\|_{\mu_{i} s_{x i}^{\prime} p, \mu_{i} s_{t i}^{\prime} p ; Q^{0}}^{\mu_{i}}
\end{aligned}
$$

где $s_{x i}, s_{t i}$ и $s_{x i}^{\prime}, s_{t i}^{\prime}$ находим из равенств

$$
p s_{x i}=q_{i}, \quad p s_{t i}=r_{i}, \quad s_{x i}^{\prime}=\frac{s_{x i}}{s_{x i}-1}, \quad s_{t i}^{\prime}=\frac{s_{t i}}{s_{t i}-1}
$$

так что

$$
s_{x i}=\frac{q_{i}}{p}, s_{t i}=\frac{r_{i}}{p}, \quad s_{x i}^{\prime}=\frac{q_{i}}{q_{i}-p}, s_{t i}^{\prime}=\frac{r_{i}}{r_{i}-p}, \quad \mu_{i} s_{x i}^{\prime} p=\frac{\mu_{i} p q_{i}}{q_{i}-p}, \mu_{i} s_{t i}^{\prime} p=\frac{\mu_{i} p r_{i}}{r_{i}-p} .
$$

Заметим, что последние два значения совпадают с $z_{x i}$ и $z_{t i}$ из леммы 1 . Продолжим неравенство (5), применяя для оценки $\left\|D^{i} u_{0}\right\|$ лемму 1 :

$$
\begin{aligned}
& \left\|u_{0}\right\|_{W\left(Q^{0}\right)} \leqslant c_{1}\left(\|b\|_{p ; Q^{0}}+\sum_{i=0}^{2 b-1}\left\|b_{i}\right\|_{q_{i}, r_{i} ; Q^{0}}\left\|D^{i} u_{0}\right\|_{z_{x i}, z_{t i} ; Q^{0}}^{\mu_{i}}\right) \\
& \leqslant c_{1}\left(\|b\|_{p ; Q^{0}}+\sum_{i=0}^{2 b-1}\left\|b_{i}\right\|_{q_{i}, r_{i} ; Q^{0}} c_{0}^{\mu_{i}}\left(\left\|u_{0}\right\|_{W\left(Q^{0}\right)}^{1 / \mu_{i}}\left\|u_{0}\right\|_{l, m ; Q^{0}}^{1-1 / \mu_{i}}+\left\|u_{0}\right\|_{\left.\left.l, m ; Q^{0}\right)^{\mu_{i}}\right)}\right)\right. \\
& \leqslant c_{1}\left(\|b\|_{p ; Q^{0}}+\sum_{i=0}^{2 b-1}\left\|b_{i}\right\|_{q_{i}, r_{i} ; Q^{0}} c_{0}^{\mu_{i}} 2^{\mu_{i}}\left(\left\|u_{0}\right\|_{W\left(Q^{0}\right)}\left\|u_{0}\right\|_{l, m ; Q^{0}}^{\mu_{i}-1}+\left\|u_{0}\right\|_{l, m ; Q^{0}}^{\mu_{i}}\right)\right) \\
& \leqslant\left\|u_{0}\right\|_{W\left(Q^{0}\right)} c_{1} \sum_{i=0}^{2 b-1}\left(2 c_{0}\right)^{\mu_{i}}\left\|b_{i}\right\|_{q_{i}, r_{i} ; Q^{0}} M^{\mu_{i}-1} \\
& \quad+c_{1}\left(\|b\|_{p ; Q^{0}}+\sum_{i=0}^{2 b-1}\left(2 c_{0}\right)^{\mu_{i}}\left\|b_{i}\right\|_{q_{i}, r_{i} ; Q^{0}} M^{\mu_{i}}\right) .
\end{aligned}
$$


В силу (4) коэффициент при $\left\|u_{0}\right\|_{W\left(Q^{0}\right)}$ не превосходит $1 / 2$, так что неравенство (6) принимает вид

$$
\left\|u_{0}\right\|_{W\left(Q^{0}\right)} \leqslant \frac{1}{2}\left\|u_{0}\right\|_{W\left(Q^{0}\right)}+\frac{1}{2} C^{0},
$$

где постоянная $C^{0}$ определяется из (6). Отсюда $\left\|u_{0}\right\|_{W\left(Q^{0}\right)} \leqslant C^{0}$.

Рассмотрим цилиндры $Q^{k-1}$ и $Q^{k}, 1 \leqslant k \leqslant K$, и допустим, что уже получена оценка $\left\|u_{0}\right\|_{W\left(Q^{k-1}\right)} \leqslant C^{k-1}$. Убедимся, что из нее следует оценка $\left\|u_{0}\right\|_{W\left(Q^{k}\right)} \leqslant C^{k}$.

Обозначим $v(x, t)=u_{0}(x, 2 k \tau-t)$ и положим

$$
\widetilde{u}(x, t)=u_{0}(x, t)-v(x, t), \quad t \in(k \tau,(k+1) \tau) .
$$

Очевидно, $\widetilde{u} \in W\left(Q^{k}\right)$ и $\widetilde{u}(x, k \tau)=0$. Рассмотрим в цилиндре $Q^{k}$ задачу

$$
\begin{gathered}
\frac{\partial u}{\partial t}+\sum_{|\alpha| \leqslant 2 b} a_{\alpha}(x, t) D^{\alpha} u=f\left(x, t, u+v, \ldots, D^{2 b-1}(u+v)\right)-\frac{\partial v}{\partial t}-\sum_{|\alpha| \leqslant 2 b} a_{\alpha}(x, t) D^{\alpha} v \\
u(x, k \tau)=0,\left.\quad D^{\omega} u\right|_{\partial Q^{k}}=0, \quad|\omega| \leqslant b-1 .
\end{gathered}
$$

Функция $\widetilde{u}$ является решением этой задачи, так как согласно предположению функция $u_{0}$ является решением исходной задачи (1). Используя оценки

$$
\|\widetilde{u}\|_{l, m ; Q^{k}} \leqslant 2\left\|u_{0}\right\|_{l, m ; Q} \leqslant 2 M
$$

и равенство $\|v\|_{W\left(Q^{k}\right)}=\left\|u_{0}\right\|_{W\left(Q^{k-1}\right)}$, аналогично (5) и (6) получаем

$$
\begin{aligned}
& \|\widetilde{u}\|_{W\left(Q^{k}\right)} \leqslant c_{1}\left\|f\left(x, t, \widetilde{u}+v, \ldots, D^{2 b-1}(\widetilde{u}+v)\right)-\frac{\partial v}{\partial t}-\sum_{|\alpha| \leqslant 2 b} a_{\alpha}(x, t) D^{\alpha} v\right\|_{p ; Q^{k}} \\
& \leqslant c_{1}\left(\|b\|_{p ; Q^{k}}+\sum_{i=0}^{2 b-1}\left\|b_{i}\right\|_{q_{i}, r_{i} ; Q^{k}}\left\|D^{i}(\widetilde{u}+v)\right\|_{z_{x i}, z_{t i} ; Q^{k}}^{\mu_{i}}+c_{2}\|v\|_{W\left(Q^{k}\right)}\right) \\
& \leqslant c_{1}\left(\|b\|_{p ; Q^{k}}+\sum_{i=0}^{2 b-1}\left\|b_{i}\right\|_{q_{i}, r_{i} ; Q^{k}} c_{0}^{\mu_{i}} 2^{\mu_{i}}\left(\|\widetilde{u}+v\|_{W\left(Q^{k}\right)}\|\widetilde{u}+v\|_{l, m ; Q^{k}}^{\mu_{i}-1}\right.\right. \\
& \left.\left.+\|\widetilde{u}+v\|_{l, m ; Q^{k}}^{\mu_{i}}\right)+c_{2}\left\|u_{0}\right\|_{W\left(Q^{k-1}\right)}\right) \\
& \leqslant\|\widetilde{u}\|_{W\left(Q^{k}\right)} c_{1} \sum_{i=0}^{2 b-1}\left(4 c_{0}\right)^{\mu_{i}} M^{\mu_{i}-1}\left\|b_{i}\right\|_{q_{i}, r_{i} ; Q^{k}} \\
& +c_{1}\left(\|b\|_{p ; Q^{k}}+\sum_{i=0}^{2 b-1}\left\|b_{i}\right\|_{q_{i}, r_{i} ; Q^{k}}\left(4 c_{0}\right)^{\mu_{i}} M^{\mu_{i}}\right) \\
& +\left\|u_{0}\right\|_{W\left(Q^{k-1}\right)} c_{1}\left(\sum_{i=0}^{2 b-1}\left(4 c_{0}\right)^{\mu_{i}}\left\|b_{i}\right\|_{q_{i}, r_{i} ; Q^{k}} M^{\mu_{i}-1}+c_{2}\right) .
\end{aligned}
$$

Так как коэффищиент при $\|\widetilde{u}\|_{W\left(Q^{k}\right)}$ не превосходит $1 / 2$, неравенство (7) принимает вид

$$
\|\widetilde{u}\|_{W\left(Q^{k}\right)} \leqslant \frac{1}{2}\|\widetilde{u}\|_{W\left(Q^{k}\right)}+c_{3}\left\|u_{0}\right\|_{W\left(Q^{k-1}\right)}+c_{4}
$$


где $c_{3}$ и $c_{4}$ определяются из (7). Отсюда

$$
\|\widetilde{u}\|_{W\left(Q^{k}\right)} \leqslant 2 c_{3}\left\|u_{0}\right\|_{W\left(Q^{k-1}\right)}+2 c_{4} .
$$

Заметим, что $u_{0}=\widetilde{u}+v$, так что $\left\|u_{0}\right\|_{W\left(Q^{k}\right)} \leqslant\|\widetilde{u}\|_{W\left(Q^{k}\right)}+\|v\|_{W\left(Q^{k}\right)}$. Используя (8) и определение функции $v$, получим

$$
\left\|u_{0}\right\|_{W\left(Q^{k}\right)} \leqslant\left(2 c_{3}+1\right)\left\|u_{0}\right\|_{W\left(Q^{k-1}\right)}+2 c_{4} \text {. }
$$

Согласно предположению $\left\|u_{0}\right\|_{W\left(Q^{k-1}\right)} \leqslant C^{k-1}$, так что из (9) следует

$$
\left\|u_{0}\right\|_{W\left(Q^{k}\right)} \leqslant C^{k}
$$

Применение индукции завершает доказательство теоремы.

3. Примеры. Рассмотрим начально-краевую задачу для уравнения второго порядка

$$
\frac{\partial u}{\partial t}-\Delta u=f(x, t, u, D u)
$$

где $f\left(x, t, \xi_{0}, \xi_{1}\right)$ - каратеодориева функция. Пусть априорная оценка $\|u\|_{\infty ; Q} \leqslant M$ известна и

$$
\left|f\left(x, t, \xi_{0}, \xi_{1}\right)\right| \leqslant b(x, t)+b_{1}(x, t)\left|\xi_{1}\right|^{\mu_{1}}
$$

где

$$
\mu_{1}=2-\frac{N+2}{p}, \quad b(x, t), b_{1}(x, t) \in L_{p}(Q), \quad p>N+2 .
$$

Тогда по теореме 1 имеем оценку

$$
\|u\|_{W_{p}^{2,1}(Q)} \leqslant C
$$

Неулучшаемость показателя $\mu_{1}$ покажем на примере задачи

$$
-\frac{\partial u}{\partial t}+\frac{\partial^{2} u}{\partial x^{2}}=b_{\varepsilon}(x, t)\left|\frac{\partial u}{\partial x}\right|^{\mu_{1}}, \quad Q=(-1,1) \times(0,1)
$$

где

$$
b_{\varepsilon}(x, t)=\frac{-\partial u_{\varepsilon} / \partial t+\partial^{2} u_{\varepsilon} / \partial x^{2}}{\left|\partial u_{\varepsilon} / \partial x\right|^{\mu_{1}}}, \quad u_{\varepsilon}(x, t)=\frac{x+t-1}{\left(x^{2}+1-t+\varepsilon\right)^{\delta}} .
$$

Пусть $\mu_{1}>2-(N+2) / p$. Можно выбрать $0<\delta<1 / 2$ такое, что $u_{\varepsilon}$ является решением этой задачи для любого $\varepsilon>0,\left\|b_{\varepsilon}\right\|_{p ; Q} \leqslant C$ и $\left\|u_{\varepsilon}\right\|_{\infty ; Q} \leqslant M$ равномерно по $\varepsilon$. Однако, $\left\|u_{\varepsilon}\right\|_{W_{p}^{2,1}(Q)} \rightarrow \infty$ при $\varepsilon \rightarrow 0$.

В качестве второго примера к теореме 1 рассмотрим уравнение

$$
\frac{\partial u}{\partial t}+(-1)^{b} \Delta^{b} u=-\sum_{i=0}^{2 b-1} \gamma_{i}(x, t, u)\left|D^{i} u\right|^{\mu_{i}}
$$

где $\gamma_{i}\left(x, t, \xi_{0}\right)$ - каратеодориевы функции,

$$
\sup _{\xi_{0} \in \mathbb{R}}\left|\gamma_{i}\left(x, t, \xi_{0}\right)\right| \in L_{p}(Q)
$$


для любого $\xi_{0} \in \mathbb{R}$ справедливо неравенство $\gamma_{i}\left(x, t, \xi_{0}\right) \cdot \xi_{0} \geqslant 0, p>N+2 b$ и

$$
\mu_{i}=\frac{1}{i+N / 2}\left(2 b+\frac{N}{2}-\frac{N+2 b}{p}\right) .
$$

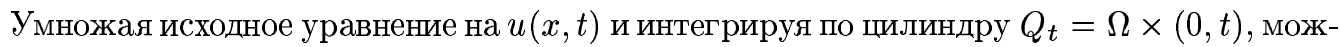
но получить для этой задачи априорную оценку $\|u\|_{2, \infty ; Q} \leqslant M$. Далее по теореме 1 получаем более сильную априорную оценку

$$
\|u\|_{W_{p}^{2 b, 1}(Q)} \leqslant C .
$$

В заключение автор выражает благодарность своему научному руководителю членкорр. РАН С.И. Похожаеву за постановку задачи и полезное обсуждение результатов, а также Я.Ш. Ильясову за ценные замечания.

\section{СПИСОК ЦИТИРОВАННОЙ ЛИТЕРАТУРЫ}

[1] Похожаев С. И. О разрешимости квазилинейных эллиптических уравнений произвольного порядка // Матем. сб. 1982. Т. 117. № 2. С. 251-265.

[2] Похожаев С. И. Об априорных оценках решений квазилинейных эллиптических уравнений произвольного порядка // Диффференц. уравнения. 1983. Т. 117. № 1. С. 101-110.

[3] Бесов О. В., Ильин В. А., Никольский С. М. Интегральные представления функций и теоремы вложения. М.: Наука, 1975.

[4] Солонников В. А. О краевых задачах для линейных параболических систем дифференциальных уравнений общего вида. Тр. МИАН. Т. 83. М.: Наука, 1965.

[5] Wahl von W. Klassische Lösbarkeit im Großen für nichtlineare parabolische Systeme und das Verhalten der Lösungen für $t \rightarrow \infty$ // Nachr. Acad. Wiss. Göttingen Math.-Phys. Kl. II. 1981. №5. P. 131-177.

[6] Wahl von W. Extention of a result of Ladyženskaja and Ural'ceva concerning second-order parabolic equations of arbitrary order // Ann. Pol. Math. 1983. V. 41. № 1. P. 63-72.

[7] Бесов О.В.Интегральные представления функций и теоремы вложения для области с условием гибкого рога // Тр. МИАН. 1984. Т. 170. С. 12-30.

[8] Колмогоров А. Н., Фомин С. В. Элементы теории функций и функционального анализа. М.: Наука, 1976. 